Оригинальная статья/Original article

УДК 664.12

DOI: http://doi.org/10.20914/2310-1202-2016-3-247-251

\begin{tabular}{c} 
Методические аспекты интегрирования технологических \\
вспомогательных средств в технологический поток производства \\
свекловичного сахара \\
\hline \hline
\end{tabular}

\begin{tabular}{lll}
\hline \hline Любовь И. Беляева, & 1 & info@rniisp.ru \\
Валентина Н. Лабузова, & \\
Алла В. Остапенко, & 1 & \\
Елена М. Скрипко & 1 & \\
\hline
\end{tabular}

технологический отдел, Российский научно-исследовательский институт сахарной промышленности, ул. Карла Маркса, 63, г. Курск, 305029, Россия

Реферат. Рассмотрены современные тенденции применения технологических вспомогательных средств в отечественной технологии свекловичного сахара. Показано, что используемые средства характеризуются высоким технологическим эффектом, пролонгированным действием, целевое их применение технологически и экономически оправдано, локальные технологии закреплены в научно-технической документации, контроль остаточных веществ средств в готовой продукции ведется по их расходу, методики определения действующих веществ в белом сахаре, жоме, мелассе отсутствуют, основная масса средств зарубежного производства. Отмечено, что эффективность и безопасность каждого средства рассматривается с позиций их узкоспециализированной направленности на локальном участке их применения, а не системно - всего технологического потока. Обоснована необходимость интегрирования технологий применения технологических вспомогательных средств в технологический поток. Обозначен спектр направлений научных исследований для создания системы интегрированных технологий применения технологических вспомогательных средств в производстве сахара, ориентированных на повышение стабильности функционирования технологического потока, безопасность готовой продукции. Разработаны исходные требования к указанной системе, предусматривающие интеграцию технологических вспомогательных средств с технологическим потоком. Сформированы методические подходы к оценке совокупного действия средств, базирующиеся на выявлении между ними синергетического или антагонистического эффектов. Приведены методические аспекты формирования структурной схемы системы интегрированных технологий, включающие декомпозицию технологического потока с выделением участка совокупного действия конкретных средств, выявление между ними синергизма-антагонизма, глубинный анализ причин проявления последнего, его нивелирование или минимизацию на основе интеграционных технологических или технических решений. Рассмотрено формирование структурной схемы интегрированной технологии на примере участка технологического потока от сгущения очищенного сока до получения утфеля I кристаллизации, на котором используются антинакипины и поверхностно-активные вещества. Отмечено, что совокупное их действие в процессе уваривания на этапе образования и роста кристаллов сахарозы сопровождается антагонистическим эффектом. Для интегрирования технологии применения антинакипина в технологический поток предусматривается использование интеграционного технологического решения - ведение процесса фильтрования сиропа через фильтровальные перегородки с диаметром пор 30-50 мкм.

Ключевые слова: технологический поток производства сахара, технологическое вспомогательное средство, интегрированная технология, совокупное действие, синергизм, антагонизм

\title{
Methodological aspects of the integration of technological processing aids in the process stream of beet sugar production
}

\begin{tabular}{lll}
\hline \hline Lyubov I. Belyaeva, & 1 & info@rniisp.ru \\
Valentina N. Labuzova, & 1 \\
Alla V. Ostapenko, & 1 \\
Elena M. Skripko & 1 & \\
\hline
\end{tabular}

1 technology department, Russian research institute of the sugar industry, Karla Marksa str., 63, Kursk, 305029, Russia

Summary. The modern trends in the application of technological processing aids in the domestic beet sugar technology were considered. It is shown that the means used are characterized by a high technological effect, prolonged action, target application of means is technologically and economically feasible, local technologies are fixed in scientific and technical documentation, control of residual compounds in the finished product is carried out at their expense, methods for determining the active substances in white sugar, pulp, molasses are not available, the bulk of foreign-made means. It is noted that each agent efficiency and safety is considered as extremely specialized orientation on the local area of application, but not in a consistent manner - the entire production flow. The necessity of integrating application technology of processing aids in technological stream. We outlined a range of research areas to create a system

Для цитирования

Беляева Л. И., Лабузова В. Н., Остапенко А. В., Скрипко Е. М. Методические аспекты интегрирования технологических вспомогательных средств в технологический поток производства свекловичного сахара // Вестник ВГУИТ. 2016. № 3. С. 247-251. doi:10.20914/2310-1202-2016-3-247-251
For citation

Belyaeva L. I., Labusowa V. N., Ostapenko A. V., Skripko E. M. Methodological aspects of the integration of technological processing aids in the process stream of beet sugar production. Vestnik VSUET [Proceedings of VSUET]. 2016. no. 3. pp. 247-251. (in Russian). doi:10.20914/2310-1202-2016-3-247-251 
of integrated process application of technological processing aids in the sugar production, focused on stability augmentation of technological flow functioning, safety of the finished product. Initial requirements for this system, providing integration of processing aids with the process stream were developed. Methodical approaches to the assessment of collective effect of means, based on the identification of synergistic or antagonistic effects were formed. Methodical aspects of formation of structures scheme of the system of integrated technologies, including technological decomposition with the release of area of collective effect of specific means, identification of synergism and antagonism, deep analysis of the causes of manifestation of the latter, its leveling or minimization based on the integration of technology or technical solutions were listed. The formation of the block diagram of integrated technology by the example of an area of the process stream from purified juice condensation to the I crystallisation massecuite reception was considered, during the process stream antiscalant and surfactants were used. It is noted that the collective effect of the process of boiling at the stage of formation and the growth of sucrose crystals was accompanied by an antagonistic effect. For integration of technology application of antiscalant in process stream it is prescribed the use of integration technological solution - establishment of filtering syrup process through a filtering partition with a pore diameter of 30-50 microns.

Keywords: process stream of sugar production, technological processing aid, integrated technology, collective effect, synergism, antagonism

\section{Введение}

В отечественном производстве свекловичного сахара реализация современных технологических решений предусматривает применение технологических вспомогательных средств (ТВC), выступающих как инструмент интенсификации технологических процессов. Ассортимент применяемых ТВС разнообразен по функциональному направлению и линейке внутри функциональных групп, что обусловлено многообразием задач локальных технологических процессов производства сахара, отличающихся сложностью, множественностью, ресурсоемкостью. Рынок ТВС успешно развивается, мотивируя сахарные заводы к активному их применению.

Анализ эволюционного развития использования ТВС в отечественной технологии сахара в течение более двух столетий в разрезе четырех условно выделенных стадий [1]: от первых классических ТВС (химических реагентов известкового молока, углекислого газа, сернистого газа) до широкого и разнообразного ассортиментного ряда средств (рисунок 1), позволяет выделить следующие основные современные тенденции их применения.

Используемые в настоящее время ТВС обладают высоким технологическим эффектом и пролонгированным действием при минимизации их расхода. Распространение получают узконаправленные препараты внутри каждой функциональной группы и многофункциональные комплексные препараты, сочетающие в себе композиции действующих веществ разных функциональных групп [2, 3].

Для каждого конкретного ТВС имеется локальная технология применения, не требующая значительных капиталовложений, позволяющая автоматически вести процесс приготовления и дозирования препарата в зависимости от параметров технологического процесса и качества обрабатываемых полуфабрикатов. Целевое применение каждого средства технологически и экономически оправдано; технологический режим и правила работы с ними закреплены в научно-технической документации (технологических инструкциях, методических рекомендациях и др.). Безопасность ТВС регулируется Техническим регламентом Таможенного союза ТР ТС 029/2012 “Требования безопасности пищевых добавок, ароматизаторов и технологических вспомогательных средств": контроль содержания остаточных количеств в готовой и побочной продукции ведется, как правило, по установленному изготовителем средства расходу, необходимому для достижения технологического эффекта. Стандартизованные методики определения действующих веществ применяемых ТВС в белом сахаре, жоме, мелассе отсутствуют. Основная масса средств - зарубежного производства: в страну импортируется до $75 \%$ пищевых ингредиентов (пищевых добавок и ТВС) [4].

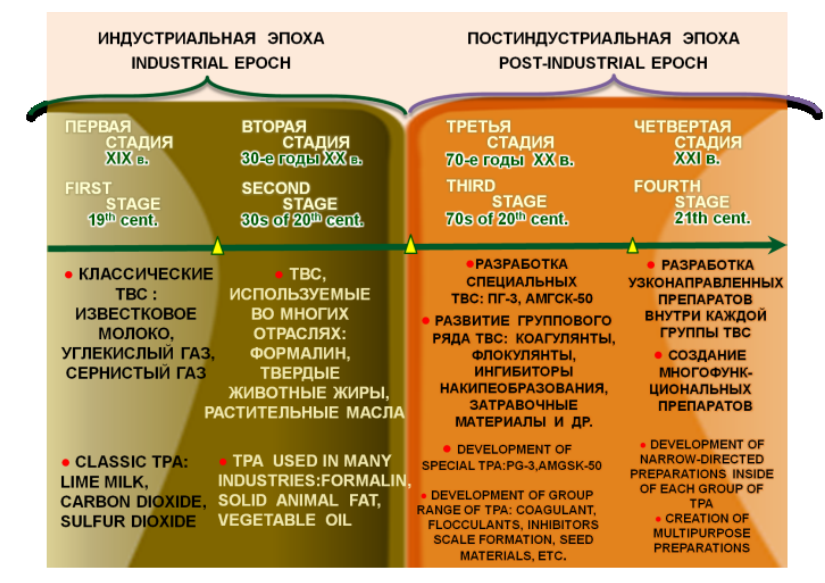

Рисунок 1. Стадии эволюции применения ТВС в производстве сахара

Figure 1. Stages of evolution of the use of the fuel assembly in the production of sugar

Однако эффективность и безопасность каждого средства рассматривается изготовителями и потребителями ТВС только с точки зрения достижения узкой технологической цели локального участка их применения. Но в условиях многовариативности использования ТВС совместное их применение в технологическом 
потоке может привести к различного рода последствиям, таким как: нивелирование заявленного технологического эффекта; возможность отрицательного действия на пищевую систему, качество протекания технологических процессов; возможность попадания остаточных количеств действующего вещества средства в сахар, жом и мелассу, которое представляет риск для здоровья людей и животных или оказывает негативное влияние на качество продукции и процессы ее производства при использовании их в качестве сырьевого ингредиента.

Вместе с тем, ТВС, как и пищевые добавки, согласно установленной международной практике, должны постоянно проходить переоценку с учетом появления новых научных данных об их воздействии на организм человека, окружающую среду, последствий их применения в пищевых системах [5]. Поэтому эффективность и безопасность каждого средства следует рассматривать системно с позиций всего сложного технологического потока производства сахара, осуществляя интегрирование локальных технологий конкретных ТВС в технологический поток. Полагаем, что четвертая стадия эволюции применения ТВС в производстве сахара завершится созданием системы интегрированных технологий применения ТВС как совокупности локальных технологий, ориентированных на повышение стабильности функционирования технологического потока. Такая система открывает новый уровень использования ТВС: дает возможность повысить эффективность единого технологического процесса производства сахара, увеличить выход и качество готовой продукции при обеспечении защиты здоровья человека и окружающей среды.

Для формирования такой системы необходимы новые знания, вытекающие из результатов научных исследований по широкому спектру направлений: разработка новых специальных ТВС; изучение поведения средств в пищевых системах сахарного производства, взаимосвязи их функциональных действий с выявлением синергизма и антагонизма; исследование совокупного влияния ТВС на качество протекания процессов, в которых они непосредственно используются, опосредованного влияния на другие процессы, изменение состава полуфабрикатов, побочной и готовой продукции, миграции действующих веществ по технологическому потоку; разработка методик определения действующих веществ в сахаре, жоме и мелассе и т. д. По части представленных направлений проводятся теоретические и экспериментальные исследования.
В результате разработаны исходные требования к системе ТВС в производстве сахара из сахарной свеклы, предусматривающие в т. ч. интеграцию ТВС с технологическим потоком, учитывающую все взаимосвязи конкретного средства в совокупном действии сдругими ТВС. Сформированы методические подходы к оценке совокупного действия ТВС при их одновременном применении в технологическом потоке [1], которые базируются на выявлении результата взаимодействия между средствами в виде антагонизма (когда одно из средств ослабляет действие другого) или синергизма (когда одно из средств усиливает действие другого). Оценка совокупного влияния ТВС предусматривает дифференциацию качества протекания процессов технологического потока в зависимости от уровня выполнения технологической задачи и состояния в них пищевой системы на основе определения репрезентативных показателей.

Полученные данные предполагается использовать в качестве основы структурной схемы интегрированных технологий применения ТВС в свеклосахарном производстве. Методические аспекты ее формирования включают выполнение декомпозиции технологического потока с выделением участков совокупного действия конкретных ТВС; выявление синергетических или антагонистических эффектов последствий совокупного действия ТВС выделенного участка; при выявлении антагонистического эффекта проводится глубинный анализ причин его проявления и предлагаются варианты интеграционных технических или технологических решений для его нивелирования или минимизации.

Рассмотрим формирование структурной схемы интегрированной технологии на примере участка технологического потока от сгущения очищенного сока до получения утфеля I кристаллизации. На указанном участке в процессах технологического потока используются функциональные группы ТВС: антинакипины - при сгущении сока; поверхностно-активные вещества (ПАВ) - при уваривании утфеля I. Антинакипин, выполнив свое функциональное назначение в процессе сгущения, в составе сиропа поступает на процесс фильтрования, где в качестве фильтровальных перегородок используются фильтровальные ткани с диаметром пор 100200 мкм, при этом около 70\% его от введенной дозы удаляется с осадком, а до 30\% мигрирует в составе фильтрованного сиропа на этап уваривания утфеля I. Соответственно, на данном этапе имеет место совокупное взаимодействие антинакипина и ПАВ (рисунок 2). 


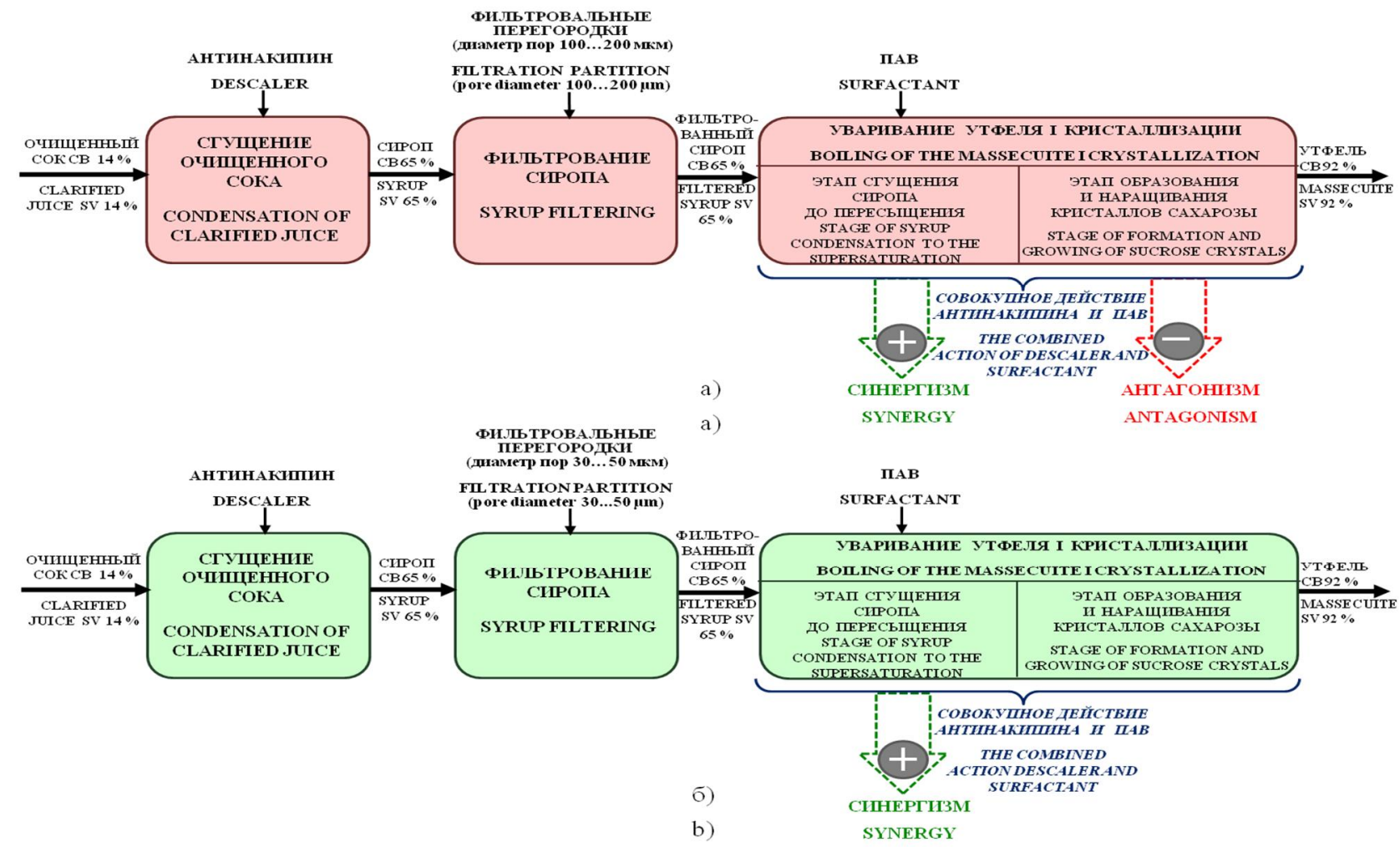

Рисунок 2. Вариант структурной схемы технологии применения антинакипина в технологическом потоке производства свекловичного сахара: а - локальной; в - интегрированной

Figure 2. Option block diagram of the application of technology in the descaling process stream of beet sugar production of a - local; $b$ - integrated

Оценив совокупное действие средств на основе вышеуказанных методических подходов, получаем объективную картину совместного взаимодействия антинакипина и ПАВ в процессе уваривания утфеля I: на этапе сгущения сиропа до пересыщения от сухих веществ $65 \%$ до $82 \%$ сухих веществ отмечается синергетический эффект, а на этапе образования и наращивания кристаллов сахарозы имеет место антагонистическое влияние антинакипина на функциональное действие ПАВ [6]. Причем, обуславливая формирование неустойчивого состояния пищевой системы утфеля I за счет ухудшения его реологических свойств, антинакипин всегда будет выступать антагонистом ПАВ.

Следовательно, для интегрирования технологии применения антинакипина в технологический поток производства сахара следует устранить или минимизировать этот эффект. Это можно выполнить путем устранения или сведения до минимума поступления антинакипина на этап уваривания утфеля I.
Указанное достигается максимальным удалением взвешенных солей кальция из сиропа, а с ними и структурно связанного антинакипина, что возможно при использовании в процессе фильтрования сиропа фильтровальных тканей с диаметром пор 30-50 мкм или фильтрующих материалов (кизельгура, перлита). Такое технологическое решение выступает как интеграционное.

Для наполнения всех позиций структурной схемы интегрированных технологий применения ТВС в свеклосахарном производстве предстоит выполнить подобные исследования по оценке совокупного действия ТВС других функциональных групп.

\section{Заключение}

Предложены методические аспекты интегрирования ТВС в технологический поток производства сахара из сахарной свеклы, реализация которых обеспечит повышение эффективности и результативности функционирования технологической линии в целом. 


\section{ЛИТЕРАТУРА}

1 Беляева Л.И. и др. Технологические вспомогательные средства в производстве сахара: эволюция применения // Сахар. 2015. № 11. С. 39-43.

2 Костенко Т.И., Кузнецова М.В., Лебедев В.С. и др. Отечественные пеногасители для сахарного производства // Сахар. 2014. № 5. С. 39-41.

3 Олянская С.П., Цырульникова В.В., Ровинский А.Д. Использование флокулянтов как метод повышения эффективности очистки диффузионного сока // Caхар. 2010. № 8. С. 43-48.

4 Никифорова Т.А. Научное обеспечение производства пищевых добавок: перспективы развития индустрии микроингредиентов в России // Материалы международной научно-практической конференции "Продовольственная безопасность и научное обеспечение развития отечественной индустрии конкурентоспособных пищевых ингредиентов”. СПб: ФГБНУ ВНИИПД, 2015. С. 150-153.

5 Commission Regulations (EU) No 257/2010 of 25 March 2010 setting up a program for the re-evaluation of approved food additives in accordance with Regulation (EC) No 1333/2008 of the European Parliament and of the Council of 16 December 2008 on food additives.

6 Беляева Л.И. и др. Совокупное влияние антинакипина и поверхностно-активного вещества на качество процесса уваривания утфеля // Материалы XIII международной научно-практической конференции "Пища. Экология. Качество". Красноярск: ФГБНУ СибНИИП, 2016. С. 140-145.

\section{СВЕДЕНИЯ ОБ АВТОРАХ}

Любовь И. Беляева к. т. н., зам. директора, Российский научно-исследовательский институт сахарной промышленности, ул. Карла Маркса, 63, г. Курск, 305029, Россия

Валентина Н. Лабузова зав. отделом, технологический отдел, Российский научно-исследовательский институг сахарной промьшленности, ул. Карла Маркса, 63, г. Курск, 305029, Россия

Алла В. Остапенко ст. науч. сотр., технологический отдел, Российский научно-исследовательский институг сахарной промышленности, ул. Карла Маркса, 63, г. Курск, 305029, Россия

Елена М. Скрипко мл. науч. сотр., технологический отдел, Российский научно-исследовательский институт сахарной промышленности, ул. Карла Маркса, 63, г. Курск, 305029, Россия

\section{КРИТЕРИЙ АВТОРСТВА}

Любовь И. Беляева предложила методику проведения теоретических и экспериментальных исследований

Алла В. Остапенко обзор литературных источников по исследуемой проблеме, провела эксперименты

Валентина Н. Лабузова консультация в ходе исследования, несёт ответственность за плагиат

Елена М. Скрипко написала рукопись, корректировала её до подачи в редакцию

\section{КОНФЛИКТ ИНТЕРЕСОВ}

Авторы заявляют об отсутствии конфликта интересов.

ПОСТУПИЛА 13.06.2016

ПРИНЯТА В ПЕЧАТЬ 23.08.2016

\section{REFERENCES}

1 Belyaeva L.I. et.al. Technological processing aids in the production of sugar: the evolution of the application. Sakhar [Sugar] 2015, no. 11, pp. 39-43. (in Russian).

2 Kostenko T.I., Kuznetsova M.V., Lebedev V.S. Domestic defoamers for sugar production. Sakhar [Sugar] 2014, no. 5, pp. 39-41. (in Russian).

3 Olanskya S.P, Tsyrulnikova V.V., Rovinskiy A.D. The use of flocculants as a method of increasing the efficiency of purification of diffusion juice. Sakhar [Sugar] 2010, no. 8, pp. 43-48. (in Russian).

4 Nikiforova T.A. Scientific support of production of food additives: prospects of development of the industry microingredients in Russia. Materialy Mezhdunarodnoy nauchno-prakticheskoy konferentcii "Prodovol'stvennaya bezopasnost' i nauchnoe obespechenie razvitiya otechestvennoj industrii konkurentnosposobnykh pishchevykh ingridientov" [Proc. Int. sci. conf. "Food safety and scientific support to the development of domestic industry competitiveness food ingredients", Saint-Petersburg] 2015, pp. 150-153. (in Russian).

5 Commission Regulations (EU) No 257/2010 of 25 March 2010 setting up a programme for the re-evaluation of approved food additives in accordance with Regulation (EC) No 1333/2008 of the European Parliament and of the Council of 16 December 2008 on food additives.

6 Belyaeva L.I. et.al. Combined effect of antiscale agents and surfactants on the quality of the process the boiling in stage. Materialy Mezhdunarodnoy nauchno-prakticheskoy konferentcii "Pisha. Ekologiy. Kachestvo" [Proc. Int. sci. conf. "Food. Ecology. Quality", Krasnoyarsk] 2016. pp. 140-145. (in Russian).

\section{INFORMATION ABOUT AUTHORS}

Lyubov I. Belyaeva candidate of technical science, deputy director, Russian research institute of the sugar industry, Karla Marksa str., 63, Kursk, 305029, Russia

Valentina N. Labusowa head of department, technology department, Russian research institute of the sugar industry, Karla Marksa str., 63, Kursk, 305029, Russia

Alla V. Ostapenko senior researcher, technology department, Russian research institute of the sugar industry, Karla Marksa str., 63, Kursk, 305029, Russia

Elena M. Skripko junior researcher, technology department, Russian research institute of the sugar industry, Karla Marksa str., 63, Kursk, 305029, Russia

\section{CONTRIBUTION}

Lyubov I. Belyaeva proposed a method of carrying out theoretical and experimental studies

Alla V. Ostapenko review of the literature on an investigated problem, conducted an experiment

Valentina N. Labusowa consultation during the study, responsible for plagiarism

Elena M. Skripko wrote the manuscript, correct it before filing in editing

\section{CONFLICT OF INTEREST}

The authors declare no conflict of interest.

RECEIVED 6.13.2016

ACCEPTED 8.23.2016 